\title{
Existence and approximation of solutions to nonlocal boundary value problems for fractional differential inclusions
}

\author{
M. Kamenskii ${ }^{1}$ V. Obukhovskii², G. Petrosyan² and Jen-Chih Yao ${ }^{3 *}$
}

\author{
"Correspondence: \\ yaojc@math.nsysu.edu.tw \\ ${ }^{3}$ Research Center for Interneural \\ Computing, China Medical \\ University Hospital, China Medical \\ University, Kaohsiung, Taiwan \\ Full list of author information is \\ available at the end of the article
}

\begin{abstract}
We study a semilinear fractional order differential inclusion in a separable Banach space $E$ of the form

$$
{ }^{C} D^{9} x(t) \in A x(t)+F(t, x(t)), \quad t \in[0, T],
$$

where ${ }^{C} D^{q}$ is the Caputo fractional derivative of order $0<q<1, A: D(A) \subset E \rightarrow E$ is a generator of a $C_{0}$-semigroup, and $F:[0, T] \times E \multimap E$ is a nonlinear multivalued map. By using the method of the generalized translation multivalued operator and a fixed point theorem for condensing multivalued maps, we prove the existence of a mild solution to this inclusion satisfying the nonlocal boundary value condition:

$$
x(0) \in \Delta(x)
$$

where $\Delta: C([0, T] ; E) \multimap E$ is a given multivalued map. The semidiscretization scheme is developed and applied to the approximation of solutions to the considered nonlocal boundary value problem.
\end{abstract}

MSC: Primary 34A45; secondary 34A08; 34G20; 47H08; 47H10; $47 \mathrm{H} 11$

Keywords: Fractional differential equation; Semilinear differential equation; Cauchy problem; Approximation; Semidiscretization; Index of the solution set; Fixed point; Condensing map; Measure of noncompactness

\section{Introduction}

In the last years, the theory of differential equations and inclusions of fractional order attracted the attention of a large number of researchers. To a large extent, this is caused by its interesting applications in physics, enginery, biology, economics, and other sciences (see, e.g., monographs [1, 4, 9, 13, 22, 26, 28, 29, 31, 35], and the references therein). It is worth noting, in this connection, that one of the most important advantages of fractional order models in comparison with those of integer order is that a fractional order derivative of a function depends on its past values and hence becomes a powerful tool for the description of memory and hereditary properties of some media. A particular advantage of such an approach appears in the investigation of nonlocal boundary value problems,

(c) The Author(s) 2019. This article is distributed under the terms of the Creative Commons Attribution 4.0 International License (http://creativecommons.org/licenses/by/4.0/), which permits unrestricted use, distribution, and reproduction in any medium, provided you give appropriate credit to the original author(s) and the source, provide a link to the Creative Commons license, and indicate if changes were made. 
that is, in the association with the differential equation or inclusion of that type an initial condition depending on the behavior of the whole solution.

In the present paper, for a semilinear fractional order differential inclusion in a separable Banach space $E$ of the form

$$
{ }^{C} D^{q} x(t) \in A x(t)+F(t, x(t)), \quad t \in[0, T],
$$

we consider the problem of existence and approximation of mild solutions to this inclusion satisfying the following nonlocal boundary value condition:

$$
x(0) \in \Delta(x) .
$$

Here ${ }^{C} D^{q}$ means the Caputo fractional derivative of order $0<q<1, A: D(A) \subset E \rightarrow E$ is a generator of a $C_{0}$-semigroup, $F:[0, T] \times E \multimap E$ is a nonlinear multivalued map, and $\Delta: C([0, T] ; E) \multimap E$ is a given multivalued map.

The above boundary condition is fairly general and includes, besides obvious cases of the Cauchy problem $\left(\Delta(x) \equiv x_{0} \in E\right)$, periodic $(\Delta(x)=x(T))$, and antiperiodic $(\Delta(x)=-x(T))$ problems, also the following particular cases:

(i) $\Delta(x)=\frac{1}{T} \int_{0}^{T} x(t) d t$ (mean value condition);

(ii) $\Delta(x)=\sum_{i=1}^{n} \alpha_{i} x\left(t_{i}\right)+\xi$, with $\xi \in E, \alpha_{i} \neq 0, t_{i} \in[0, T], i=1, \ldots, n$ (multipoint discrete mean condition);

(iii) $\Delta(x) \equiv \mathcal{M}$, with $\mathcal{M} \subset E$ being a prescribed set (the generalized Cauchy problem).

Among a large amount of papers dedicated to fractional-order equations and inclusions in Banach spaces, let us mention [3, 5, 15-17, 20, 21, 23, 24, 27, 33, 34, 36], where various existence results were obtained. In particular, in [5] a technique based on the weak topology methods was used to study a semilinear fractional differential inclusion subjected to a nonlocal initial condition. Notice that the results on the existence of solutions to the Cauchy and the periodic problems for semilinear differential inclusions in a Banach space were obtained in the authors' papers $[15,17]$ by applying the methods of the theory of condensing multivalued maps. In [16] and [25] the authors justified the scheme of semidiscretization of the Cauchy problem for differential equations of the same type and presented results on the approximation of solutions to this problem. Notice also that the semidiscretization method for initial and periodic problems of ODEs in a Banach space was studied in $[6,11,12,30,32]$, among other works. In the present work, we develop and extend the investigations in the same direction.

The structure of the paper is as follows. In the next section, we recall necessary notions and facts from the theory of differential equations of fractional order, measures of noncompactness and condensing maps. In the third section, we introduce the translation multivalued operator along the trajectories of the problem under consideration and prove that it is condensing with respect of the Hausdorff measure of noncompactness (Theorem 3). Based on this result, we show that this multivalued operator has a fixed point and therefore our problem has a solution (Theorem 4). In the last section, we develop the semidiscretization scheme and apply it to justify the approximation of solutions to the considered nonlocal boundary value problem (Theorem 6). 


\section{Preliminaries}

\subsection{Differential equations of fractional order}

Recall some notions and definitions, which we will need in the sequel (details can be found, e.g., in $[22,28,29,35])$.

Let $E$ be a real Banach space.

Definition 1 The Riemann-Liouville fractional derivative of order $q \in(0,1)$ of a continuous function $g:[0, a] \rightarrow E$ is the function $D^{q} g$ given by

$$
D^{q} g(t)=\frac{1}{\Gamma(1-q)} \frac{d}{d t} \int_{0}^{t}(t-s)^{-q} g(s) d s,
$$

provided the right-hand side of this equality is well defined.

Here $\Gamma$ is the Euler gamma-function defined by

$$
\Gamma(r)=\int_{0}^{\infty} s^{r-1} e^{-s} d s
$$

Definition 2 The Caputo fractional derivative of order $q \in(0,1)$ of a continuous function $g:[0, a] \rightarrow E$ is the function ${ }^{C} D^{q} g$ defined by

$$
{ }^{C} D^{q} g(t)=\left(D^{q}(g(\cdot)-g(0))\right)(t)
$$

provided the right-hand side of this equality is well defined.

Definition 3 A function of the form

$$
E_{\alpha, \beta}(z)=\sum_{n=0}^{\infty} \frac{z^{n}}{\Gamma(\alpha n+\beta)}, \quad \alpha, \beta>0, z \in \mathbb{C},
$$

is called the Mittag-Leffler function.

Denote $E_{q, 1}$ by $E_{q}$. Notice that from the relations (see, e.g., [33])

$$
E_{q}(-z)=\int_{0}^{\infty} \xi_{q}(\theta) e^{-z \theta} d \theta
$$

and

$$
E_{q, q}(-z)=\int_{0}^{\infty} q \theta \xi_{q}(\theta) e^{-z \theta} d \theta
$$

where

$$
\begin{aligned}
& \xi_{q}(\theta)=\frac{1}{q} \theta^{-1-\frac{1}{q}} \Psi_{q}\left(\theta^{-1 / q}\right), \\
& \Psi_{q}(\theta)=\frac{1}{\pi} \sum_{n=1}^{\infty}(-1)^{n-1} \theta^{-q n-1} \frac{\Gamma(n q+1)}{n !} \sin (n \pi q), \quad \theta \in \mathbb{R}_{+},
\end{aligned}
$$


it follows that

$$
E_{q}(\tau)>0, \quad E_{q, q}(\tau)>0 \quad \text { for } \tau<0 .
$$

Consider a scalar Cauchy problem, i.e., an equation of the form

$$
{ }^{C} D^{q} x(t)=\lambda x(t)+f(t), \quad t \in[0, T],
$$

with the initial condition

$$
x(0)=x_{0},
$$

where $\lambda \in \mathbb{R}, f:[0, T] \rightarrow \mathbb{R}$ is a continuous function. By a solution of this problem we mean a continuous function $x:[0, T] \rightarrow \mathbb{R}$ satisfying condition (2.5) whose fractional derivative ${ }^{C} D^{q} x$ is also continuous and satisfies equation (2.4). It is known (see [22], Example 4.9) that the unique solution of this equation has the form

$$
x(t)=E_{q}\left(\lambda t^{q}\right) x_{0}+\int_{0}^{t}(t-s)^{q-1} E_{q, q}\left(\lambda(t-s)^{q}\right) f(s) d s .
$$

\subsection{Measures of noncompactness and condensing maps}

Let $\mathcal{E}$ be a Banach space. Introduce the following notation:

- $\operatorname{Pb}(\mathcal{E})=\{A \subseteq \mathcal{E}: A \neq \emptyset$ is bounded $\}$

- $\operatorname{Pv}(\mathcal{E})=\{A \in \operatorname{Pb}(\mathcal{E}): A$ is convex $\}$;

- $K(\mathcal{E})=\{A \in \operatorname{Pb}(\mathcal{E}): A$ is compact $\}$;

- $K v(\mathcal{E})=\operatorname{Pv}(\mathcal{E}) \cap K(\mathcal{E})$.

Definition 4 (see, e.g., $[2,18])$ Let $(\mathcal{A}, \geq)$ be a partially ordered set. A function $\beta: \mathrm{Pb}(\mathcal{E}) \rightarrow$ $\mathcal{A}$ is called the measure of noncompactness (MNC) in $\mathcal{E}$ if for each $\Omega \in \mathrm{Pb}(\mathcal{E})$ we have

$$
\beta(\overline{\operatorname{co}} \Omega)=\beta(\Omega),
$$

where $\overline{\mathrm{co}} \Omega$ denotes the closure of the convex hull of $\Omega$.

A measure of noncompactness $\beta$ is called:

(1) monotone if for each $\Omega_{0}, \Omega_{1} \in \mathrm{Pb}(\mathcal{E}), \Omega_{0} \subseteq \Omega_{1}$ implies $\beta\left(\Omega_{0}\right) \leq \beta\left(\Omega_{1}\right)$;

(2) nonsingular if for each $a \in \mathcal{E}$ and each $\Omega \in \operatorname{Pb}(\mathcal{E})$, we have $\beta(\{a\} \cup \Omega)=\beta(\Omega)$;

If $\mathcal{A}$ is a cone in a Banach space generating a partial order $\geq$, then the MNC $\beta$ is called:

(3) regular if $\beta(\Omega)=0$ is equivalent to the relative compactness of $\Omega \in \mathrm{Pb}(\mathcal{E})$;

(4) real if $\mathcal{A}$ is the set of all real numbers $\mathbb{R}$ with the natural ordering;

(5) algebraically semiadditive if $\beta\left(\Omega_{0}+\Omega_{1}\right) \leq \beta\left(\Omega_{0}\right)+\beta\left(\Omega_{1}\right)$ for every $\Omega_{0}, \Omega_{1} \in \mathrm{Pb}(\mathcal{E})$.

As the example of a real MNC obeying all above properties, we can consider the Hausdorff $\operatorname{MNC} \chi(\Omega)$ :

$$
\chi(\Omega)=\inf \{\varepsilon>0 \text {, for which } \Omega \text { has a finite } \varepsilon \text {-net in } \mathcal{E}\} .
$$


Notice that the Hausdorff MNC satisfies the semi-homogeneity condition, i.e.,

$$
\chi(\lambda \Omega)=|\lambda| \chi(\Omega),
$$

for every $\lambda \in \mathbb{R}$ and $\Omega \in \mathrm{P}(\mathcal{E})$. More generally, if $\mathcal{L}: \mathcal{E} \rightarrow \mathcal{E}$ is a bounded linear operator then

$$
\chi(\mathcal{L}(\Omega))=\|\mathcal{L}\| \chi(\Omega)
$$

for every $\Omega \in \mathrm{P}(\mathcal{E})$ (see, e.g., [2]).

Recall that the norm of a set $M \in \mathrm{Pb}(\mathcal{E})$ is defined by the formula:

$$
\|M\|=\sup _{x \in M}\|x\|_{\mathcal{E}}
$$

Definition 5 (see, e.g., $[7,10,18])$ Let $X$ be a metric space. A multivalued map $\mathcal{F}: X \rightarrow$ $P(\mathcal{E})$ is called upper semicontinuous (u.s.c.) if

$$
\mathcal{F}^{-1}(V)=\{x \in X: \mathcal{F}(x) \subset V\}
$$

is an open subset of $X$ for each open set $V \subset \mathcal{E}$;

Definition 6 (see, e.g., $[10,18]$ ) A multivalued map $\mathcal{F}: X \rightarrow P(\mathcal{E})$ is said to be a Vietoris multivalued map, provided there exists a metric space $X^{\prime}$ and a pair of continuous maps $t: X^{\prime} \rightarrow X$ and $r: X^{\prime} \rightarrow \mathcal{E}$ such that:

(i) $t$ is proper, i.e., $t^{-1}(K)$ is compact for every compact $K \subset X$;

(ii) for each $x \in X$ the set $t^{-1}(x)$ is acyclic, i.e., it has the same homologies as a one-point space;

(iii) $\mathcal{F}(x)=r\left(t^{-1}(x)\right), \forall x \in X$.

The class of Vietoris multivalued maps is sufficiently broad. To demonstrate this, recall the following notions.

Definition 7 A metric space $X$ is called contractible if there exist a point $x_{0} \in X$ and a continuous map (homotopy) $h:[0,1] \times X \rightarrow X$ such that $h(0, x)=x$ and $h(1, x)=x_{0}$ for all $x \in X$.

It is obvious that convex and, more generally, star-shaped sets are contractible.

Definition 8 (see [14]) A compact metric space $A$ is called an $R_{\delta}$-set if there exists a decreasing sequence $\left\{A_{n}\right\}$ of compact contractible sets such that

$$
A=\bigcap_{n \geq 1} A_{n}
$$

Notice that an $R_{\delta}$-set is acyclic, but need not be contractible (see an example in [10]).

Definition 9 Let $X$ be a metric space, $\mathcal{E}$ a Banach space. A u.s.c. multivalued map $\mathcal{F}$ : $X \rightarrow K(\mathcal{E})$ is called an $R_{\delta}$-multivalued map if for every $x \in X$ the set $\mathcal{F}(x)$ is $R_{\delta}$. 
It is easy to see that an $R_{\delta}$-multivalued map is a Vietoris map. In fact, as $t$ and $r$ we may take natural projections from the graph $\Gamma_{\mathcal{F}} \subset X \times \mathcal{E}$

$$
t: \Gamma_{\mathcal{F}} \rightarrow X, \quad r: \Gamma_{\mathcal{F}} \rightarrow \mathcal{E}
$$

Definition 10 A multivalued map $\mathcal{F}: X \rightarrow K(\mathcal{E})$ is called an $R_{\delta}^{c}$-multivalued map if there exists metric spaces $X_{0}=X, X_{1}, \ldots, X_{k}, X_{k+1}=\mathcal{E}$ and $R_{\delta}$-multivalued maps $\mathcal{F}_{i}: X_{i} \rightarrow$ $K\left(X_{i+1}\right), i=0, \ldots, k$ such that $\mathcal{F}$ may be represented as the composition:

$$
\mathcal{F}=\mathcal{F}_{k} \circ F_{k-1} \circ \cdots \circ \mathcal{F}_{0}
$$

From Proposition 3.4.1(a) of [18] it follows that every $R_{\delta}^{c}$-multivalued map is a Vietoris map.

Then, by applying Corollary 3.4.3 in [18], we get the following fixed point theorem, which we will need in the sequel.

Theorem 1 Let $\mathcal{M}$ be a convex, closed and bounded subset of a Banach space $\mathcal{E}$, and $\mathcal{F}: \mathcal{M} \rightarrow K(\mathcal{M})$ an $R_{\delta}^{c}$-multivalued map which is $(k, \chi)$-condensing for some $0 \leq k<1$, i.e.,

$$
\chi(\mathcal{F}(\Omega)) \leq k \chi(\Omega)
$$

for every $\Omega \subset \mathcal{M}$. Then there exists a point $x_{*} \in \mathcal{M}$ such that $x_{*} \in \mathcal{F}\left(x_{*}\right)$.

Recall some notions (see, e.g., $[7,18])$. Let $E$ be a Banach space.

Definition 11 For a given $1 \leq p \leq \infty$, a multivalued function $G:[0, \tau] \rightarrow K(E)$ is called:

- $L^{p}$-integrable if it admits an $L^{p}$-Bochner integrable selection, i.e., there exists a function $g \in L^{p}((0, \tau) ; E)$ such that $g(t) \in G(t)$ for a.e. $t \in[0, \tau]$;

- $L^{p}$-integrably bounded if there exists a function $\xi \in L^{p}((0, \tau))$ such that

$$
\|G(t)\| \leq \xi(t)
$$

for a.e. $t \in[0, \tau]$.

The set of all $L^{p}$-integrable selections of a multivalued function $G:[0, \tau] \rightarrow K(E)$ is denoted by $\mathcal{S}_{G}^{p}[0, \tau]$.

Definition 12 The integral of an $L^{p}$-integrable multivalued function $G:[0, \tau] \rightarrow K(E)$ is defined by

$$
\int_{0}^{\tau} G(s) d s=\left\{\int_{0}^{\tau} f(s) d s: f \in \mathcal{S}_{G}^{p}[0, \tau]\right\} .
$$

In the sequel we will need the following important property on the $\chi$-estimation of the integral of a multivalued function. 
Lemma 1 (see Theorem 4.2.3 in [18]) Let E be a separable Banach space and $G:[0, \tau] \rightarrow$ $K(E)$ an integrable, integrably-bounded multivalued function such that

$$
\chi(G(t)) \leq v(t) \text { for a.e. } t \in[0, \tau]
$$

where $\chi$ is the Hausdorff MNC in E and $v(\cdot) \in L_{+}^{1}(0, \tau)$. Then

$$
\chi\left(\int_{0}^{\tau} G(s) d s\right) \leq \int_{0}^{\tau} v(s) d s .
$$

We will need the following auxiliary assertion which is an analogue of the known Gronwall lemma on integral inequalities.

Lemma 2 ([17], Lemma 13) Let a bounded measurable function $\omega:[0, T] \rightarrow \mathbb{R}$ satisfy the integral inequality

$$
\omega(t) \leq E_{q}\left(-\eta t^{q}\right) \omega(0)+\int_{0}^{t}(t-s)^{q-1} E_{q, q}\left(-\eta(t-s)^{q}\right)(K+k \omega(s)) d s
$$

where $K \geq 0,0<k<\eta$. Then

$$
\omega(t) \leq E_{q}\left((-\eta+k) t^{q}\right) \omega(0)+K \int_{0}^{t}(t-s)^{q} E_{q, q}\left((-\eta+k)(t-s)^{q}\right) d s .
$$

\section{Existence result}

For a semilinear fractional order differential inclusion in a separable Banach space $E$ of the form

$$
{ }^{C} D^{q} x(t) \in A x(t)+F(t, x(t)), \quad t \in[0, T],
$$

consider the problem of existence of mild solutions to this inclusion satisfying the following boundary value condition:

$$
x(0) \in \Delta(x)
$$

under the following basic assumptions.

As earlier, the symbol ${ }^{C} D^{q} x$ denotes the Caputo fractional derivative of order $q \in(0,1)$. Everywhere in the sequel we suppose that the linear operator $A$ satisfies condition

(A) $A: D(A) \subseteq E \rightarrow E$ is a linear closed (not necessarily bounded) operator generating a bounded $C_{0}$-semigroup $\{U(t)\}_{t \geq 0}$ of linear operators in $E$.

We will assume that a nonlinear multivalued map $F:[0, T] \times E \rightarrow K v(E)$ obeys the following conditions:

$(F 1)$ for each $x \in E$ the multivalued function $F(\cdot, x):[0, T] \rightarrow K v(E)$ admits a measurable selection;

$(F 2)$ for a.e. $t \in[0, T]$ the multivalued map $F(t, \cdot): E \rightarrow K v(E)$ is u.s.c.;

(F3) there exists a function $\alpha \in L_{+}^{\infty}([0, T])$ such that

$$
\|F(t, x)\|_{E} \leq \alpha(t)\left(1+\|x(t)\|_{E}\right) \quad \text { for a.e. } t \in[0, T]
$$


(F4) there exists a function $\mu \in L^{\infty}([0, T])$ such that for each bounded set $\Omega \subset E$ we have

$$
\chi(F(t, \Omega)) \leq \mu(t) \chi(\Omega)
$$

for a.e. $t \in[0, T]$, where $\chi$ is the Hausdorff MNC in $E$.

We will assume that an $R_{\delta}^{c}$-multivalued map $\Delta: C([0, T] ; E) \rightarrow K(E)$ obeys the following conditions:

$(\Delta 1)$ there exists a functional $\mathfrak{f}: C([0, T] ; \mathbb{R}) \rightarrow \mathbb{R}_{+}$and a constant $C \geq 0$ which is:

(i) sublinear in the sense that $\mathfrak{f}\left(\lambda_{0} \psi_{0}+\lambda_{1} \psi_{1}\right) \leq \lambda_{0} \mathfrak{f}\left(\psi_{0}\right)+\lambda_{1} \mathfrak{f}\left(\psi_{1}\right), \forall \lambda_{0} \geq 0$, $\lambda_{1} \geq 0, \psi_{0}, \psi_{1} \in C([0, T] ; \mathbb{R})$;

(ii) monotone nondecreasing in the sense that $\psi_{0}, \psi_{1} \in C([0, T] ; \mathbb{R})$, $\psi_{0}(t) \leq \psi_{1}(t), \forall t \in[0, T]$ implies $\mathfrak{f}\left(\psi_{0}\right) \leq \mathfrak{f}\left(\psi_{1}\right)$

such that for all $x \in C([0, T] ; E)$

$$
\|\Delta(x)\|_{E} \leq \mathfrak{f}\left(\|x(\cdot)\|_{E}\right)+C
$$

$(\Delta 2)$ Let $\Omega \subset C([0, T] ; E)$ be a nonempty bounded set and $x$ a solution of scalar problem (2.4) $-(2.5)$ with $\lambda=-\eta, \eta>0$ and $x_{0}=\chi(\Omega(0))$ such that

$$
\chi(\Omega(t)) \leq x(t), \quad \forall t \in[0, T] .
$$

Then

$$
\chi(\Delta(\Omega)) \leq \mathfrak{f}(x) .
$$

Remark 1 For the particular cases of the boundary conditions (i)-(iii) from the introduction section, the functional $\mathfrak{f}$ has the form

(i) $\mathfrak{f}(\varphi)=\frac{1}{T} \int_{0}^{T}|\varphi(s)| d s$;

(ii) $\mathfrak{f}(\varphi)=\sum_{i=1}^{n}\left|\alpha_{i}\right|\left|\varphi\left(t_{i}\right)\right|, \alpha_{i} \neq 0, t_{i} \in[0, T], i=1, \ldots, n$;

(iii) $\mathfrak{f}(\varphi)=0$.

For a given $x \in C([0, T] ; E)$, consider the multivalued function

$$
\Phi_{x}:[0, T] \rightarrow K v(E), \quad \Phi_{x}(t)=F(t, x(t)) .
$$

From the above conditions $(F 1)-(F 3)$ it follows (see, e.g., [18], Theorem 1.3.5) that the multivalued function $\Phi_{x}$ is $L^{p}$-integrable for each $p \geq 1$.

To solve our problem, we will use the superposition multivalued operator $\mathcal{P}_{F}^{\infty}$ : $C([0, T] ; E) \multimap L^{\infty}([0, T] ; E)$ defined by

$$
\mathcal{P}_{F}^{\infty}(x)=\mathcal{S}_{\Phi_{x}}^{\infty} .
$$

Definition 13 (see, e.g., [15]) A mild solution to the Cauchy problem for inclusion (3.1) with initial condition

$$
x(0)=x_{0} \in E
$$


on an interval $[0, T]$ is a function $x \in C([0, T] ; E)$ which can be represented as

$$
x(t)=\mathcal{G}(t) x_{0}+\int_{0}^{t}(t-s)^{q-1} \mathcal{T}(t-s) \phi(s) d s, \quad t \in[0, T]
$$

where $\phi \in \mathcal{P}_{F}^{\infty}(x)$,

$$
\begin{aligned}
& \mathcal{G}(t)=\int_{0}^{\infty} \xi_{q}(\theta) U\left(t^{q} \theta\right) d \theta, \\
& \mathcal{T}(t)=q \int_{0}^{\infty} \theta \xi_{q}(\theta) U\left(t^{q} \theta\right) d \theta,
\end{aligned}
$$

and the function $\xi_{q}(\theta)$ is defined by (2.1)-(2.2).

Remark 2 (see, e.g. $[34,35]) \xi_{q}(\theta) \geq 0, \int_{0}^{\infty} \xi_{q}(\theta) d \theta=1, \int_{0}^{\infty} \theta \xi_{q}(\theta) d \theta=\frac{1}{\Gamma(q+1)}$.

Lemma 3 (see [34, 35]) The operator functions $\mathcal{G}$ and $\mathcal{T}$ possess the following properties:

(1) for each $t \in[0, T], \mathcal{G}(t)$ and $\mathcal{T}(t)$ are linear bounded operators, more precisely, for each $x \in E$ we have

$$
\begin{aligned}
\|\mathcal{G}(t) x\|_{E} & \leq M\|x\|_{E}, \\
\|\mathcal{T}(t) x\|_{E} & \leq \frac{q M}{\Gamma(1+q)}\|x\|_{E},
\end{aligned}
$$

where

$$
M=\sup _{t \geq 0}\|U(t)\|
$$

(2) the operator functions $\mathcal{G}(\cdot)$ and $\mathcal{T}(\cdot)$ are strongly continuous, i.e., functions $t \in[0, T] \rightarrow \mathcal{G}(t) x$ and $t \in[0, T] \rightarrow \mathcal{T}(t) x$ are continuous for each $x \in E$.

Remark 3 Comparing formula (3.4) of a mild solution with (2.6), we get in a scalar case with $\eta>0$ :

$$
E_{q}\left(-\eta t^{q}\right)=\mathcal{G}(t), \quad E_{q, q}\left(-\eta t^{q}\right)=\mathcal{T}(t), \quad t \in[0, T]
$$

Then, taking into account that in this case $U(t)=e^{-\eta t}$, from (3.5) and (3.6) we have the following estimates:

$$
\begin{aligned}
& E_{q}\left(-\eta t^{q}\right) \leq 1, \quad t \in[0, T] \\
& E_{q, q}\left(-\eta t^{q}\right) \leq \frac{q}{\Gamma(1+q)}, \quad t \in[0, T]
\end{aligned}
$$

By the symbol $\Sigma_{x_{0}}^{F}$ we will denote the set of all mild solutions to the Cauchy problem (3.1), (3.3) on the interval $[0, T]$.

From the results of $[15,17]$ about the existence and topological structure of solutions to the Cauchy problem (3.1) and (3.3), the next assertion follows. 
Theorem 2 Under conditions $(A),(F 1)-(F 4)$, the solution set $\Sigma_{x_{0}}^{F}$ is an $R_{\delta}$-set and, moreover, the multivalued map $\Sigma: E \rightarrow C([0, T] ; E)$, defined as

$$
\Sigma(x)=\Sigma_{x}^{F}
$$

is u.s.c. and hence an $R_{\delta}$-multivalued map.

Now we will consider the translation multivalued operator $\Theta: D \subseteq E \multimap E$ along the trajectories of problem (3.1)-(3.2) defined as $\Theta=\Delta \circ \Sigma$, where $D \subset E$ is an open subset. It is clear that $\Theta$ is an $R_{\delta}^{c}$-multivalued map.

Theorem 3 Under assumptions $(A),(F 1)-(F 4)$, suppose additionally that

(A1) the semigroup $U$ is exponentially decreasing in the sense that

$$
\begin{gathered}
\|U(t)\| \leq e^{-\eta t}, \quad t \geq 0 \\
\text { for some } \eta>\|\mu\|_{\infty} \text {, where } \mu(\cdot) \text { is the function from condition (F4). } \\
k:=\mathfrak{f}(\xi)<1,
\end{gathered}
$$

where $\xi(t)=E_{q}\left(\left(-\eta+\|\mu\|_{\infty}\right) t^{q}\right)$, then the translation multivalued operator $\Theta$ is $(k, \chi)$ condensing with respect to the Hausdorff MNC $\chi$ in $E$.

Proof Let $\Omega \subset D$ is a nonempty bounded set. For $0 \leq t \leq T$ consider the set

$$
\Sigma(\Omega)(t)=\left\{v(t): v \in \Sigma_{x}^{F}[0, T], x \in \Omega\right\} .
$$

It is clear that $\Sigma(\Omega)(0)=\Omega$ and

$$
\Sigma(\Omega)(t) \subseteq \mathcal{G}(t) \Omega+\int_{0}^{t}(t-s)^{q-1} \mathcal{T}(t-s) F(s, \Sigma(\Omega)(s)) d s, \quad 0 \leq t \leq T,
$$

from where, by using property ( $F 3)$, we get for $0 \leq t \leq T$ :

$$
\begin{aligned}
\|\Sigma(\Omega)(t)\| & \leq\|\mathcal{G}(t)\|\|\Omega\|+\int_{0}^{t}(t s)^{q-1}\|\mathcal{T}(t-s)\|\|F(s, \Sigma(\Omega)(s))\| d s \\
& \leq\|\mathcal{G}(t)\|\|\Omega\|+\int_{0}^{t} \alpha(s)(t-s)^{q-1}\|\mathcal{T}(t-s)\|(1+\| \Sigma(\Omega)(s)) \| d s .
\end{aligned}
$$

By using the estimates (see [3])

$$
\begin{gathered}
\|\mathcal{G}(t)\| \leq E_{q}\left(-\eta t^{q}\right), \\
\|\mathcal{T}(t)\| \leq E_{q \cdot q}\left(-\eta t^{q}\right),
\end{gathered}
$$

we have

$$
\begin{aligned}
\|\Sigma(\Omega)(t)\| \leq & E_{q}\left(-\eta t^{q}\right)\|\Omega\| \\
& +\int_{0}^{t} \alpha(s)(t-s)^{q-1} E_{q, q}\left(-\eta(t-s)^{q}\right)(1+\|\Sigma(\Omega)(s)\|) d s .
\end{aligned}
$$


Applying estimates (3.7)-(3.8), we get

$$
\|\Sigma(\Omega)(t)\| \leq\|\Omega\|+\frac{q}{\Gamma(1+q)} \int_{0}^{t} \alpha(s)(t-s)^{q-1}(1+\|\Sigma(\Omega)(s)\|) d s,
$$

from where, by using Gronwall inequality, we conclude that the set $\Sigma(\Omega)$ is bounded.

Then by Theorem 4.2.4 of [18] the function

$$
t \in[0, T] \rightarrow \chi(\Sigma(\Omega)(t))
$$

is measurable.

Applying the properties of the MNC $\chi$ (see Sect. 2.2), we get the following estimates:

$$
\begin{aligned}
\chi(\Sigma(\Omega)(t)) & \leq \chi\left(\mathcal{G}(t) \Omega+\int_{0}^{t}(t-s)^{q-1} \mathcal{T}(t-s) F(s, \Sigma(\Omega)(s)) d s\right) \\
& \leq\|\mathcal{G}(t)\| \chi(\Omega)+\int_{0}^{t}(t-s)^{q-1}\|\mathcal{T}(t-s)\|\|\mu\|_{\infty} \chi(\Sigma(\Omega)(s)) d s .
\end{aligned}
$$

By using estimates (3.9)-(3.10), we obtain

$$
\begin{aligned}
\chi(\Sigma(\Omega)(t)) \leq & E_{q}\left(-\eta t^{q}\right) \chi(\Sigma(\Omega)(0)) \\
& +\int_{0}^{t}(t-s)^{q-1} E_{q, q}\left(-\eta(t-s)^{q}\right)\|\mu\|_{\infty} \chi(\Sigma(\Omega)(s)) d s .
\end{aligned}
$$

Applying Lemma 2 with $K=0$, we get

$$
\chi(\Sigma(\Omega)(t)) \leq E_{q}\left(\left(-\eta+\|\mu\|_{\infty}\right) t^{q}\right) \chi(\Omega) .
$$

Now by using condition $(\Delta 2)$ and denoting $\xi(t)=E_{q}\left(\left(-\eta+\|\mu\|_{\infty}\right) t^{q}\right)$, we obtain

$$
\chi(\Delta \circ \Sigma(\Omega)) \leq \mathfrak{f}(\xi \chi(\Omega)) \leq \mathfrak{f}(\xi) \chi(\Omega)=k \chi(\Omega)
$$

giving the claim.

Now we are in position to prove the main result of this section.

Theorem 4 Under conditions $(A),(A 1)$, and $(F 1)-(F 4)$, let

$$
\eta>\lambda,
$$

where $\eta$ is the constant from condition (A1) and $\lambda=\max \left\{\|\mu\|_{\infty},\|\alpha\|_{\infty}\right\}$ with functions $\alpha(\cdot)$ and $\mu(\cdot)$ from conditions (F3) and (F4), respectively. If

$$
k:=\mathfrak{f}(\xi)<1,
$$

where $\xi(t)=E_{q}\left((-\eta+\lambda) t^{q}\right)$, then problem (3.1)-(3.2) has a solution. 
Proof For an arbitrary $x_{0} \in E$, take a function $x \in \Sigma_{x_{0}}^{F}$. Then for every $t \in[0, T]$ we have

$$
x(t) \in \mathcal{G}(t) x_{0}+\int_{0}^{t}(t-s)^{q-1} \mathcal{T}(t-s) F\left(s, \Sigma_{x_{0}}^{F}(s)\right) d s .
$$

Consider a continuous function $\rho:[0, T] \rightarrow \mathbb{R}$ given as

$$
\rho(t)=\left\|\Sigma_{x_{0}}^{F}(t)\right\|_{E}
$$

Then we have the following estimates:

$$
\begin{aligned}
\rho(t) & \leq\left\|\mathcal{G}(t) x_{0}+\int_{0}^{t}(t-s)^{q-1} \mathcal{T}(t-s) F\left(s, \Sigma_{x_{0}}^{F}(s)\right) d s\right\|_{E} \\
& \leq\|\mathcal{G}(t)\|\left\|x_{0}\right\|_{E}+\int_{0}^{t}(t-s)^{q-1}\|\mathcal{T}(t-s)\|\|\alpha\|_{\infty}(1+\rho(s)) d s .
\end{aligned}
$$

Applying estimates (3.9)-(3.10), we get

$$
\rho(t) \leq E_{q}\left(-\eta t^{q}\right) \rho(0)+\int_{0}^{t}(t-s)^{q-1} E_{q, q}\left(-\eta(t-s)^{q}\right)\|\alpha\|_{\infty}(1+\rho(s)) d s .
$$

By using Lemma 2, we obtain

$$
\begin{aligned}
\rho(t) & \leq E_{q}\left((-\eta+\lambda) t^{q}\right) \rho(0)+\|\alpha\|_{\infty} \int_{0}^{t}(t-s)^{q-1} E_{q, q}\left((-\eta+\lambda)(t-s)^{q}\right) d s \\
& =\left\|x_{0}\right\|_{E} E_{q}\left((-\eta+\lambda) t^{q}\right)+\mathcal{C},
\end{aligned}
$$

where

$$
\mathcal{C}=\|\alpha\|_{\infty} \int_{0}^{t}(t-s)^{q-1} E_{q, q}\left((-\eta+\lambda)(t-s)^{q}\right) d s
$$

is a constant.

Let $b(t)=\left\|x_{0}\right\|_{E} E_{q}\left((-\eta+\lambda) t^{q}\right)+\mathcal{C}$.

Now, by using condition $(\Delta 1)$, we have

$$
\|\Delta(x)\|_{E} \leq \mathfrak{f}\left(\|x(\cdot)\|_{E}\right)+C \leq \mathfrak{f}(\rho)+C \leq \mathfrak{f}(b(\cdot))+C \leq\left\|x_{0}\right\|_{E} \mathfrak{f}(\xi)+\mathfrak{f}(\mathcal{C})+C .
$$

So, if we take

$$
R \geq \frac{\mathfrak{f}(\mathcal{C})+C}{1-\mathfrak{f}(\xi)},
$$

then $\left\|x_{0}\right\|_{E} \leq R$ obviously implies $\Delta(x) \leq R$.

This means that the translation multivalued operator $\Theta$ transforms the ball $B_{R}(0) \subset E$ into itself, and hence, by Theorem 1 , it has a fixed point $x_{*} \in B_{R}(0)$. Therefore there exists a function $\widetilde{x}(\cdot) \in \Sigma_{x_{*}}^{F}$ such that

$$
\widetilde{x}(0) \in \Delta(\widetilde{x}) .
$$




\section{Approximation of solutions}

In this section we will apply a semidiscretization scheme for the approximation of solutions to problem (3.1)-(3.2).

Along with inclusion (3.1), for a given sequence of positive numbers $\left\{h_{n}\right\}$ converging to zero, consider the inclusions

$$
D^{q} x_{h}(t) \in A_{h} x_{h}(t)+F_{h}\left(t, x_{h}(t)\right), \quad t \in[0, T],
$$

where $h \in H=\overline{\left\{h_{n}\right\}}$ is the semidiscretization parameter, $A_{h}: D\left(A_{h}\right) \subset E_{h} \rightarrow E_{h}$ are closed linear operators in Banach spaces $E_{h}$ generating bounded $C_{0}$-semigroups $\left\{U_{h}(t)\right\}_{t \geq 0}$. We assume $E_{0}=E, A_{0}=A, F_{0}=F$ and suppose that multivalued maps $F_{h}:[0, T] \times E_{h} \rightarrow K v\left(E_{h}\right)$ satisfy conditions of type (F1)-(F4) for each $h \in H$ with the functions $\alpha$ and $\mu$ not depending on $h$.

We suppose that for each $h \in H$ there exist linear operators $Q_{h}: E_{h} \rightarrow E, Q_{0}=I$, and $P_{h}: E \rightarrow E_{h}, P_{0}=I$ such that

$$
P_{h} Q_{h}=I_{h},
$$

where $I_{h}$ is the identity on $E_{h}$ and

$$
Q_{h} P_{h} x \rightarrow x
$$

as $h \rightarrow 0$ for each $x \in E$. We suppose that the operators $P_{h}$ and $Q_{h}$ are uniformly bounded

$$
\left\|P_{h}\right\| \leq 1, \quad\left\|Q_{h}\right\| \leq 1
$$

for all $h \in H$.

The nonlocal boundary value condition for inclusion (4.1) will be considered of the following form:

$$
x_{h}(0) \in P_{h} \Delta\left(Q_{h} x_{h}(\cdot)\right) .
$$

For inclusion (4.1), besides condition (4.5), we will need the initial condition of the form

$$
x_{h}(0)=y_{0}^{h}
$$

where $y_{0}^{h} \in E_{h}$. The set of mild solutions to problem (4.1), (4.6) in the space $C\left([0, T] ; E_{h}\right)$ will be denoted as $\Sigma_{y_{0}^{h}}^{F_{h}}$.

Consider the integral equation

$$
x_{h}(t)=\mathcal{G}_{h}(t) x_{h}(0)+\int_{0}^{t}(t-s)^{q-1} \mathcal{T}_{h}(t-s) f_{h}(s) d s, \quad t \in[0, T],
$$

where $f_{h} \in \mathcal{P}_{F_{h}}^{\infty}\left(x_{h}\right)$ and the operator functions $\mathcal{G}_{h}$ and $\mathcal{T}_{h}$ are defined similarly as in Definition 13:

$$
\mathcal{G}_{h}(t)=\int_{0}^{\infty} \xi_{q}(\theta) U_{h}\left(t^{q} \theta\right) d \theta, \quad \mathcal{T}_{h}(t)=q \int_{0}^{\infty} \theta \xi_{q}(\theta) U_{h}\left(t^{q} \theta\right) d \theta .
$$


Notice that a function $\bar{x}_{h} \in C\left([0, T] ; E_{h}\right)$ is a solution of Eq. (4.7) if and only if it is a mild solution of problem (4.1), (4.6) with $y_{0}^{h}=\bar{x}_{h}(0)$.

We will assume that

(H1) for each $x \in E$,

$$
Q_{h} U_{h}(t) P_{h} x \rightarrow U(t) x
$$

as $h \rightarrow 0$ uniformly in $t \in[0, T]$;

$(H 2)$ there exists $k>0$ such that

$$
\chi_{E}\left(\bigcup_{h \in H} Q_{h} F_{h}\left(t, P_{h}(\Omega)\right)\right) \leq k \chi_{E}(\Omega)
$$

for each $t \in[0, T]$ and bounded $\Omega \subset E$.

We suppose also that

(H3) the multivalued map $(h, x) \multimap Q_{h} F_{h}\left(t, P_{h} x\right)$ is u.s.c. for a.e. $t \in[0, T]$.

Remark 4 Hypothesis $(H 1)$ may be equivalently formulated in terms of the strong convergence of the resolvents in the following way:

$$
Q_{h}\left(A_{h}+\lambda I\right)^{-1} P_{h} x \rightarrow(A+\lambda I)^{-1} x, \quad \forall x \in E .
$$

This is an analogue of the Trotter-Kato theorem (see, e.g., [19], Chap. IX, Theorem 2.16 or [25], Theorem 2.6).

In the sequel we will need the following assertion.

Lemma 4 ([16], Lemma 2) For each $x \in E$, we have the following relations:

$$
\begin{aligned}
Q_{h} \mathcal{G}_{h}(t) P_{h} x & \rightarrow \mathcal{G}(t) x, \\
Q_{h} \mathcal{T}_{h}(t) P_{h} x & \rightarrow \mathcal{T}(t) x,
\end{aligned}
$$

as $h \rightarrow 0$ uniformly in $t \in[0, T]$.

Consider the multivalued map $G: H \times C([0, T] ; E) \multimap C([0, T] ; E)$ defined by the equality

$$
G(h, x)(t)=\left\{y: y(t)=Q_{h} \mathcal{G}_{h}(t) P_{h} x(0)+\int_{0}^{t}(t-s)^{q-1} Q_{h} \mathcal{T}_{h}(t-s) f_{h}(s) d s\right\},
$$

with $f_{h} \in \mathcal{P}_{F_{h}}^{\infty}\left(P_{h} x\right)$.

Notice that solutions $x_{h}$ of inclusion (4.1) and fixed points of $G(h, \cdot)$ are connected in the following way: if $\bar{x} \in C([0, T] ; E)$ is a fixed point of the multivalued map $G(h, \cdot)$ then the function $\bar{x}_{h} \in C\left([0, T] ; E_{h}\right)$ defined by the formula

$$
\bar{x}_{h}=P_{h} \bar{x}
$$

is the solution of equation (4.7) and, hence of problem (4.1), (4.6) with $y_{0}=P_{h} \bar{x}(0)$. Conversely, if $\bar{x}_{h}$ is a solution of equation (4.7) and hence of problem (4.1), (4.6) with $y_{0}^{h}=\bar{x}_{h}(0)$ then $Q_{h} \bar{x}_{h}$ is a fixed point of the multivalued map $G(h, \cdot)$. 
Under above conditions, for each $h \in H$, there exists the translation multivalued operator $\Theta^{h}: D_{h} \subset E_{h} \multimap E_{h}$ along the trajectories of (4.1)-(4.5) defined as

$$
\Theta^{h}(x)=\left\{z \in P_{h} \Delta\left(Q_{h} x_{h}(\cdot)\right): x_{h} \in \Sigma_{P_{h} x}^{F_{h}}\right\}
$$

where $D_{h} \subset E_{h}$ is an open subset.

Consider the family of multivalued maps $\Gamma: H \times E \rightarrow E$

$$
\Gamma(h, x)=Q_{h} \Theta^{h} P_{h} x, \quad x \in E .
$$

Theorem 5 Under the above conditions, suppose additionally that

$\left(A 1_{h}\right)$ the semigroups $U_{h}$ are exponentially decreasing in the sense that

$$
\begin{gathered}
\left\|U_{h}(t)\right\| \leq e^{-\eta t}, \quad t \geq 0 \\
\text { for some } \eta>k \text {, where } k \text { is the constant from condition }(H 2) . \\
m:=\mathfrak{f}(\zeta)<1
\end{gathered}
$$

where $\zeta(t)=E_{q}\left((-\eta+k) t^{q}\right)$, then the family $\Gamma$ is $\left(m, \chi_{E}\right)$-condensing in the sense that for every nonempty bounded set $\Omega \subset E$ we have

$$
\chi_{E}(\Gamma(H \times \Omega)) \leq m \chi_{E}(\Omega)
$$

Proof Let $\Omega \subset E$ be a nonempty bounded set.

For $h \in H$ and $0 \leq t \leq T$ consider the set

$$
\Sigma_{h}(\Omega)(t)=\left\{y(t): y \in \Sigma_{x^{h}}^{F_{h}}, x^{h}=P_{h} x, x \in \Omega\right\} .
$$

It is clear that $Q_{h} \Sigma_{h}(\Omega)(0)=Q_{h} P_{h}(\Omega)$ and for each $0 \leq t \leq T$ we have

$$
\bigcup_{h \in H} Q_{h} \Sigma_{h}(\Omega)(t) \subseteq \bigcup_{h \in H}\left(Q_{h} \mathcal{G}_{h}(t) P_{h} \Omega+\int_{0}^{t}(t-s)^{q-1} Q_{h} \mathcal{T}_{h}(t-s) F_{h}\left(s, P_{h}(\Omega)(s)\right) d s\right)
$$

Similarly to what was done while proving Theorem 3, one can show that the set $\bigcup_{h \in H} Q_{h} \Sigma_{h}(\Omega)[0, T] \subset C([0, T] ; E)$ is bounded and then, by virtue of Theorem 4.2.4 of [18], the function

$$
t \in[0, T] \rightarrow \chi\left(\bigcup_{h \in H} Q_{h} \Sigma_{h}(\Omega)(t)\right)
$$

is measurable and evidently bounded. 
Applying the properties of the MNC $\chi$ (see Sect. 2) and (H2), (4.4) we get the following estimates:

$$
\begin{aligned}
\chi & \left(\bigcup_{h \in H} Q_{h} \Sigma_{h}(\Omega)(t)\right) \\
\leq & \chi\left(\bigcup_{h \in H}\left(Q_{h} \mathcal{G}_{h}(t) P_{h} \Omega+\int_{0}^{t}(t-s)^{q-1} Q_{h} \mathcal{T}_{h}(t-s) P_{h} Q_{h} F_{h}\left(s, P_{h} Q_{h} \Sigma_{h}(\Omega)(s)\right) d s\right)\right) \\
\leq & \sup _{h \in H}\left(\left\|Q_{h}\right\|\left\|\mathcal{G}_{h}(t)\right\|\left\|P_{h}\right\| \chi(\Omega)\right. \\
& \left.+\int_{0}^{t}(t-s)^{q-1}\left\|Q_{h}\right\|\left\|\mathcal{T}_{h}(t-s)\right\|\left\|P_{h}\right\| \chi\left(\bigcup_{h \in H} Q_{h} F_{h}\left(s, P_{h} Q_{h} \Sigma_{h}(\Omega)(s)\right)\right) d s\right) \\
\leq & \sup _{h \in H}\left(\left\|\mathcal{G}_{h}(t)\right\| \chi(\Omega)+\int_{0}^{t}(t-s)^{q-1}\left\|\mathcal{T}_{h}(t-s)\right\| k \chi\left(\bigcup_{h \in H} Q_{h} \Sigma_{h}(\Omega)(s)\right) d s\right) .
\end{aligned}
$$

Applying estimates (3.9)-(3.10), we have

$$
\begin{aligned}
\chi\left(\bigcup_{h \in H} Q_{h} \Sigma_{h}(\Omega)(t)\right) \\
\leq E_{q}\left(-\eta t^{q}\right) \chi\left(\bigcup_{h \in H} Q_{h} P_{h} \Sigma_{h}(\Omega)(0)\right) \\
\quad+\int_{0}^{t}(t-s)^{q-1} E_{q, q}\left(-\eta(t-s)^{q}\right) k \chi\left(\bigcup_{h \in H} Q_{h} \Sigma_{h}(\Omega)(s)\right) d s .
\end{aligned}
$$

By using Lemma 2 with $K=0$, we get

$$
\chi\left(\bigcup_{h \in H} Q_{h} \Sigma_{h}(\Omega)(t)\right) \leq E_{q}\left((-\eta+m) t^{q}\right) \chi(\Omega) .
$$

Applying condition $(\Delta 2)$, we have

$$
\chi(\Gamma(H \times \Omega))=\chi\left(\bigcup_{h \in H} \Delta Q_{h} \Sigma_{h}(\Omega)\right) \leq \mathfrak{f}(\zeta) \chi(\Omega)=m \chi(\Omega) .
$$

Consider now fixed points

$$
x^{h} \in \Gamma\left(h, x^{h}\right)
$$

of the multivalued map $\Gamma(h, x)$ in the ball $B_{R}(0)$, where $R$ satisfies inequality (3.13). Notice that then the points $P_{h} x^{h}$ will be initial values for solutions of the approximate problems (4.1), (4.6) (with $\left.y_{0}^{h}=P_{h} x^{h}\right)$. Consider a sequence $\left\{x^{h_{n}}\right\}$ with $h_{n} \rightarrow 0$.

Theorem 6 Under condition (3.12), the sequence $\left\{x^{h_{n}}\right\}$ is relatively compact and its limit points are initial values of solutions to inclusion (3.1) satisfying boundary value condition (3.2). 
Proof Notice that by Theorem 5 we have

$$
\chi\left(\left\{\bar{x}^{h_{n}}\right\}\right) \leq m \chi\left(\left\{x^{h_{n}}\right\}\right)
$$

implying, since $m<1$, that $\chi\left(\left\{x^{h_{n}}\right\}\right)=0$, and hence the sequence $\left\{x^{h_{n}}\right\}$ is relatively compact. Consider solutions $\kappa^{h_{n}}$ of inclusion (4.1) satisfying the boundary value condition

$$
\kappa^{h_{n}}(0)=P_{h_{n}} x^{h_{n}}
$$

Then the functions $v^{h_{n}}=Q_{h_{n}} \kappa^{h_{n}}$ are fixed points of the multivalued map $G\left(h_{n}, \cdot\right)$, where $G: H \times C([0, T] ; E) \multimap C([0, T] ; E)$ is defined as

$$
G(h, v)=\left\{y: y(t)=Q_{h} \mathcal{G}_{h}(t) \kappa^{h_{h}}(0)+\int_{0}^{t}(t-s)^{q-1} Q_{h} \mathcal{T}_{h}(t-s) f_{h}(s) d s\right\}
$$

where $f_{h} \in \mathcal{P}_{Q_{h} F_{h} P_{h}}^{\infty}\left(v^{h}\right)$ and, moreover,

$$
v^{h_{n}}(0) \in \Delta\left(v^{h_{n}}\right)
$$

which is equivalent to

$$
\kappa^{h_{n}}(0) \in P_{h_{n}} \Delta\left(Q_{h_{n}} \kappa^{h_{n}}\right)
$$

From the properties of the MNC $\chi$, estimates (3.12), (4.4) and (3.9)-(3.10), which are valid for $\mathcal{G}_{h}$ and $\mathcal{T}_{h}$ for all $h \in H$, the next estimate follows:

$$
\chi\left(\left\{v^{h_{n}}(t)\right\}\right) \leq \int_{0}^{t}(t-s)^{q-1} E_{q, q}\left(-\eta(t-s)^{q}\right)\|\mu\|_{\infty} \chi\left(v^{h_{n}}(s)\right) d s
$$

Applying Lemma 2, we get

$$
\chi\left(\left\{v^{h_{n}}(t)\right\}\right) \equiv 0, \quad t \in[0, T] .
$$

Let us show now that the functions $\left\{v^{h_{n}}\right\}$ are equicontinuous on the interval $[0, T]$. By (2) of Lemma 3 the functions $\left\{\mathcal{G}(\cdot) x^{h_{n}}\right\}$ are equicontinuous. Applying property (4.8) and the compactness of the sequence $\left\{x^{h_{n}}\right\}$, we conclude that the functions $\left\{Q_{h_{n}} \mathcal{G}_{h n}(\cdot) P_{h_{n}} x^{h_{n}}\right\}$ are equicontinuous.

It means that now it is sufficient to show the equicontinuity of the functions $\left\{v^{h_{n}}\right\}$ given by the equalities

$$
v^{h_{n}}(t)=\int_{0}^{t}(t-s)^{q-1} Q_{h_{n}} \mathcal{T}_{h_{n}}(t-s) P_{h_{n}} f_{h_{n}}(s) d s
$$

where $f_{h_{n}} \in \mathcal{P}_{Q_{h_{n}} F_{h_{n}} P_{h_{n}}}^{\infty}\left(v^{h_{n}}\right)$.

By (4.13) we have

$$
\chi\left(\left\{f_{h_{n}}(s)\right\}\right)=0 \quad \text { a.e. } s \in[0, T] .
$$


From condition $(F 3)$ applied to $F_{h_{n}}$, we get the uniform boundedness estimate

$$
\left\|f_{h_{n}}\right\|_{L^{\infty}} \leq C
$$

where $C>0$ is a certain constant.

Now, for a given $\varepsilon>0$ choose $d>0$ such that

$$
\frac{C d^{q}}{\Gamma(1+q)}<\varepsilon
$$

Then

$$
\left\|\int_{t-d}^{t}(t-s)^{q-1} Q_{h_{n}} \mathcal{T}_{h_{n}}(t-s) P_{h_{n}} f_{h_{n}}(s) d s\right\|<\varepsilon
$$

It remains to demonstrate only the equicontinuity of the functions $\left\{w^{h_{n}}\right\}$ defined for $t>d$ as

$$
w^{h_{n}}(t)=\int_{0}^{t-d}(t-s)^{q-1} Q_{h_{n}} \mathcal{T}_{h_{n}}(t-s) P_{h_{n}} f_{h_{n}}(s) d s
$$

By Theorem 4.2.5 of [18], for a given $0<\delta<T$ there exist a measurable subset $m_{\delta} \subset[0, T-$ $\delta$ ], meas $\left(m_{\delta}\right)<\delta$, and a compact set $K_{\delta} \subset E$ such that

$$
f_{h_{n}}(s) \in W_{\delta}\left(K_{\delta}\right) \quad \text { a.e. } s \in[0, T-\delta] \backslash m_{\delta},
$$

where $W_{\delta}$ denotes the $\delta$-neighborhood of the set $K_{\delta}$. But then

$$
\begin{aligned}
w^{h_{n}}(t)= & \int_{[0, t-d] \cap m_{\delta}}(t-s)^{q-1} Q_{h_{n}} \mathcal{T}_{h_{n}}(t-s) P_{h_{n}} f_{h_{n}}(s) d s \\
& +\int_{[0, t-d] \backslash m_{\delta}}(t-s)^{q-1} Q_{h_{n}} \mathcal{T}_{h_{n}}(t-s) P_{h_{n}} f_{h_{n}}(s) d s .
\end{aligned}
$$

Notice that the first term in this sum may be estimated in norm by the value

$$
\frac{d^{q-1} C}{\Gamma(1+q)} \delta
$$

which can be made less than $\varepsilon$ under an appropriate choice of $\delta$.

To demonstrate the equicontinuity of the second term with respect to $h_{n}$ notice that, by (4.9), for any $t_{1}, t_{2} \in[0, T], t_{1}<t_{2}, t_{2}-t_{1}<\gamma$ we will have

$$
\left\|\left(t_{2}-s\right)^{q-1} Q_{h_{n}} \mathcal{T}_{h_{n}}\left(t_{2}-s\right) P_{h_{n}} f_{h_{n}}(s)-\left(t_{1}-s\right)^{q-1} Q_{h_{n}} \mathcal{T}_{h_{n}}\left(t_{1}-s\right) P_{h_{n}} f_{h_{n}}(s)\right\|<\varepsilon,
$$

provided $\gamma$ is sufficiently small. Therefore

$$
\begin{aligned}
& \int_{\left[0, t_{1}-d\right] \backslash m_{\delta}}\left\|\left(t_{2}-s\right)^{q-1} Q_{h_{n}} \mathcal{T}_{h_{n}}\left(t_{2}-s\right) P_{h_{n}} f_{h_{n}}(s)-\left(t_{1}-s\right)^{q-1} Q_{h_{n}} \mathcal{T}_{h_{n}}\left(t_{1}-s\right) P_{h_{n}} f_{h_{n}}(s)\right\| d s \\
& \quad<\varepsilon\left(t_{1}-d\right)<\varepsilon T .
\end{aligned}
$$


At last,

$$
\int_{t_{1}-d}^{t_{2}-d}\left\|\left(t_{2}-s\right)^{q-1} Q_{h_{n}} \mathcal{T}_{h_{n}}\left(t_{2}-s\right) P_{h_{n}} f_{h_{n}}(s)\right\| d s \leq \frac{C}{q}\left(d^{q}-(d-\gamma)^{q}\right)<\varepsilon,
$$

provided $\gamma>0$ is taken sufficiently small.

So, the sequence $\left\{v^{h_{n}}\right\}$ is relatively compact in $C([0, T] ; E)$. We will assume, w.l.o.g., that $v^{h_{n}} \rightarrow v^{0}$.

Now, let a subsequence of $\left\{x^{h_{n}}\right\}$, denoted as the whole sequence, converge to $x^{0}$. We will show that $v_{0}$ is a solution of inclusion (3.1) with the initial condition

$$
v^{0}(0)=x^{0}
$$

In fact, from assumption (H2) it follows that for selectors $f_{h_{n}}$ of the superposition multivalued opeator $\mathcal{P}_{Q_{h_{n}} F_{h_{n}} P_{h_{n}}}^{\infty}\left(v^{h_{n}}\right)$ relation (4.15) is true. This means that the sequence $\left\{f_{h_{n}}\right\}$ satisfies the Diestel condition (see [8]) and hence it is weakly compact in $L^{1}((0, T) ; E)$. Without loss of generality, we assume that $f_{h_{n}} \rightarrow f_{0}$. Then, applying standard reasonings based on the Mazur lemma (see, e.g., [18]), one can come to the conclusion that there exists a sequence of convex combinations of functions $f_{h_{n}}$,

$$
\widetilde{f}_{i}=\sum_{n=i}^{\infty} \epsilon_{i n} f_{h_{n}}
$$

which converges a.e. on $(0, T)$ to a function $\widetilde{f}_{0}$ such that

$$
\tilde{f}_{0}(t) \in F\left(v_{0}(t)\right) \text { a.e. } t \in[0, T] .
$$

Applying the Lebesgue convergence theorem and properties (4.8)-(4.9), we can pass to the limit as $n \rightarrow \infty$ in the equality

$$
v^{h_{n}}(t)=Q_{h_{n}} \mathcal{G}_{h_{n}}(t) P_{h_{n}} x^{h_{n}}+\int_{0}^{t}(t-s)^{q-1} Q_{h_{n}} \mathcal{T}_{h_{n}}(t-s) P_{h_{n}} f_{h_{n}}(s) d s
$$

and obtain

$$
\nu^{0}(t)=\mathcal{G}(t) x^{0}+\int_{0}^{t}(t-s)^{q-1} \mathcal{T}(t-s) \widetilde{f}_{0}(s) d s
$$

proving that the function $v^{0}$ is a solution of (3.1) with the initial condition (4.16). Passing to the limit in inclusion (4.12), we get

$$
v^{0}(0)=x^{0} \in \Delta\left(v^{0}\right)
$$

which concludes the proof. 


\section{Funding}

The work is supported by the joint Taiwan MOST—Russia RFBR grant 17-51-52022. The work of the first, second and third authors is supported by the Ministry of Education and Science of the Russian Federation in the frameworks of the project part of the state work quota (Project No 1.3464.2017/4.6). J.C. Yao was supported by the Grant MOST 106-2923-E-039-001-MY3.

Abbreviations

Not applicable.

Availability of data and materials

Data sharing not applicable to this article as no datasets were generated or analyzed during the current study.

\section{Competing interests}

The authors declare that they have no competing interests.

\section{Authors' contributions}

All authors contributed equally and significantly in writing this article. All authors read and approved the final manuscript.

\section{Author details}

${ }^{1}$ Faculty of Mathematics, Voronezh State University, Voronezh, Russia. ${ }^{2}$ Faculty of Physics and Mathematics, Voronezh State Pedagogical University, Voronezh, Russia. ${ }^{3}$ Research Center for Interneural Computing, China Medical University Hospital, China Medical University, Kaohsiung, Taiwan.

\section{Publisher's Note}

Springer Nature remains neutral with regard to jurisdictional claims in published maps and institutional affiliations.

Received: 22 November 2018 Accepted: 21 December 2018 Published online: 21 January 2019

\section{References}

1. Abbas, S., Benchohra, M., N'Guerekata, G.M.: Topics in Fractional Differential Equations, Developments in Mathematics. Springer, New York (2012)

2. Ahmerov, R.R., Kamenskii, M.I., Potapov, A.S., Rodkina, A.E., Sadowskii, B.N.: Measures of Noncompactness and Condensing Operators. Birkhäuser, Boston (1992)

3. Anh, C.T., Ke, T.D.: On nonlocal problems for retarded fractional differential equations in Banach spaces. Fixed Point Theory 15(2), 373-392 (2014)

4. Baleanu, D., Diethelm, K., Scalas, E., Trujillo, J.J.: Fractional Calculus Models and Numerical Methods. World Scientific, New York (2012)

5. Benedetti, I., Obukhovskii, V., Taddei, V.: On generalized boundary value problems for a class of fractional differential inclusions. Fract. Calc. Appl. Anal. 20(6), 1424-1446 (2017)

6. Bobylev, N.A., Kim, J.K., Korovin, S.K., Piskarev, S.: Semidiscrete approximations of semilinear periodic problems in Banach spaces. Nonlinear Anal. 33(5), 473-482 (1998)

7. Borisovich, Yu.G., Gelman, B.D., Myshkis, A.D., Obukhovskii, V.V.: Introduction to the Theory of Multivalued Maps and Differential Inclusions, 2nd edn. Librokom, Moscow (2011) (in Russian)

8. Diestel, J., Ruess, W.M., Schachermayer, W.: Weak compactness in $L^{1}(\mu$, X). Proc. Am. Math. Soc. 118, 447-453 (1993)

9. Diethelm, K.: The Analysis of Fractional Differential Equations. Springer, Berlin (2010)

10. Górniewicz, L.: Topological Fixed Point Theory of Multivalued Mappings. Topological Fixed Point Theory and Its Applications, vol. 4, 2nd edn. Springer, Dordrecht (2006)

11. Gurova, I.N.: On semidiscretization methods for quasilinear equations with a noncompact semigroup. Russ. Math. (Izv. VUZ) 44(4), 58-63 (2000)

12. Gurova, I.N., Kamenskii, M.I.: On the semidiscretization method in the problem of periodic solutions of quasilinear autonomous parabolic equations. Differ. Equ. 32(1), 106-112 (1996)

13. Hilfer, R: Applications of Fractional Calculus in Physics. World Scientific, Singapore (2000)

14. Hyman, D.H.: On decreasing sequences of compact absolute retracts. Fundam. Math. 64, 91-97 (1969)

15. Kamenskii, M., Obukhovskii, V., Petrosyan, G., Yao, J.-C.: On semilinear fractional order differential inclusions in Banach spaces. Fixed Point Theory 18(1), 269-292 (2017)

16. Kamenskii, M., Obukhovskii, V., Petrosyan, G., Yao, J.-C.: On approximate solutions for a class of semilinear fractional-order differential equations in Banach spaces. Fixed Point Theory Appl. 2017, 28 (2017)

17. Kamenskii, M., Obukhovskii, V., Petrosyan, G., Yao, J.-C.: Boundary value problems for semilinear differential inclusions of fractional order in a Banach space. Appl. Anal. 97(4), 571-591 (2018)

18. Kamenskii, M., Obukhovskii, V., Zecca, P.: Condensing Multivalued Maps and Semilinear Differential Inclusions in Banach Spaces. de Gruyter Series in Nonlinear Analysis and Applications, vol. 7. de Gruyter, Berlin (2001)

19. Kato, T.: Perturbation Theory for Linear Operators. Springer, Berlin (1980)

20. Ke, T.D., Loi, N.V., Obukhovskii, V.: Decay solutions for a class of fractional differential variational inequalities. Fract. Calc. Appl. Anal. 18(3), 531-553 (2015)

21. Ke, T.D., Obukhovskii, V., Wong, N.-C., Yao, J.-C.: On a class of fractional order differential inclusions with infinite delays. Appl. Anal. 92(1), 115-137 (2013)

22. Kilbas, A.A., Srivastava, H.M., Trujillo, J.J.: Theory and Applications of Fractional Differential Equations. North-Holland Mathematics Studies, vol. 204. Elsevier, Amsterdam (2006)

23. Lakshmikantham, $V:$ : Theory of fractional functional differential equations. Nonlinear Anal. 69(10), 3337-3343 (2008)

24. Lakshmikantham, V., Vatsala, A.S.: Basic theory of fractional differential equations. Nonlinear Anal. 69(8), 2677-2682 (2008) 
25. Liu, R., Li, M., Piskarev, S.: Approximation of semilinear fractional Cauchy problem. Comput. Methods Appl. Math. 15(2), 203-212 (2015)

26. Miller, K.S., Ross, B.: An Introduction to the Fractional Calculus and Fractional Differential Equations. Wiley, New York (1993)

27. Obukhovskii, V., Yao, J.-C:: Some existence results for fractional functional differential equations. Fixed Point Theory $11(1), 85-96(2010)$

28. Podlubny, l.: Fractional Differential Equations. Academic Press, San Diego (1999)

29. Samko, S.G., Kilbas, A.A., Marichev, O.I.: Fractional Integrals and Derivatives, Theory and Applications. Gordon \& Breach, Yverdon (1993)

30. Stuart, A.: Convergence and stability in the numerical approximation of dynamical systems. In: The State of the Art in Numerical Analysis, York, 1996. Inst. Math. Appl. Conf. Ser. New Ser., vol. 63, pp. 145-169. Oxford University Press, New York (1997)

31. Tarasov, V.E.: Fractional Dynamics. Applications of Fractional Calculus to Dynamics of Particles, Fields and Media. Nonlinear Physical Science. Springer, Heidelberg; Higher Education Press, Beijing (2010)

32. Vainikko, G.: Approximative methods for nonlinear equations (two approaches to the convergence problem). Nonlinear Anal. 2(6), 647-687 (1978)

33. Wang, R.-N., Chen, D.-H., Xiao, T.-J.: Abstract fractional Cauchy problems with almost sectorial operators. J. Differ. Equ. 252(1), 202-235 (2012)

34. Zhang, Z., Liu, B.: Existence of mild solutions for fractional evolution equations. Fixed Point Theory 15(1), 325-334 (2014)

35. Zhou, Y:: Fractional Evolution Equations and Inclusions: Analysis and Control. Elsevier, London (2016)

36. Zhou, Y., Jiao, F.: Existence of mild solutions for fractional neutral evolution equations. Comput. Math. Appl. 59(3), 1063-1077 (2010)

\section{Submit your manuscript to a SpringerOpen ${ }^{\circ}$ journal and benefit from:}

- Convenient online submission

- Rigorous peer review

- Open access: articles freely available online

- High visibility within the field

- Retaining the copyright to your article

Submit your next manuscript at $\boldsymbol{\nabla}$ springeropen.com 